\title{
Cognitive Aspects of Students' Mathematical Reasoning Habits: A Study on Utilizing Lesson Study and Open Approach
}

\author{
Thong-oon Manmai, Maitree Inprasitha* and Narumon Changsri
}

Faculty of Education, Khon Kaen University, Khon Kaen 40002, Thailand

\begin{abstract}
This study aimed to investigate the cognitive aspects of the mathematical reasoning habits of $3^{\text {rd }}$ Grade students by utilizing Lesson Study (LS) and Open Approach (OA) in teaching mathematics. The cognitive aspects of mathematical reasoning habits are a powerful complement to teaching mathematics because they are particularly focused on skills and procedures. However, the major problem with teaching mathematical reasoning is that teachers cannot teach mathematical reasoning habits directly but can set up tasks that encourage reasoning. A total of eight students were purposively selected as they have been taught using OA treatment in the LS process for two years, from 2016 to 2018 . Four study instruments were used: lesson plans, activity worksheets, field notes, and an interview protocol. In addition, the National Council of Teachers of Mathematics (NCTM) (2009) framework, consisting of four cognitive aspects of mathematical reasoning habits, namely (i) analyzing a problem; (ii) implementing a strategy; (iii) using connections, and (iv) reflecting on a solution, was employed to determine the cognitive aspects of mathematical reasoning habits. Qualitative results revealed three pieces of evidence to support all four cognitive aspects of mathematical reasoning habits. In conclusion, the results of this study contribute significantly to our recognition

ARTICLE INFO

Article history:

Received: 1 June 2021

Accepted: 19 August 2021

Published: 8 December 2021

DOI: https://doi.org/10.47836/pjssh.29.4.27

E-mail addresses:

thongoon_m@kkumail.com (Thong-oon Manmai)

inprasitha_crme@kku.ac.th (Maitree Inprasitha)

changsri_crme@kku.ac.th (Narumon Changsri) of the importance of OA treatment in the LS process while teaching mathematics in improving students' mathematical reasoning habits.

*Corresponding author 


\section{INTRODUCTION}

Mathematical reasoning is one of the core mathematical learning components, and informed learning skills and innovations are recognized as ways to enhance human quality and potential in the $21^{\text {st }}$-Century (Oslington et al., 2020). Classroom teaching is a cultural activity that involves social interaction between two or more parties, namely the teacher and his or her students and between students, and subject matter is an essential element in the social interaction (Lim et al., 2013). For some time, the teachers' beliefs have been identified as a challenge for the reform of mathematics instruction (Stipek et al., 2001). Mathematical reasoning, or the principles of mathematical reasoning, is part of the mathematical thinking process that enables students to enhance their mathematical skills. Consequently, mathematical reasoning could be thought of as the 'glue' which helps students make sense of mathematics (Askew, 2020). Askew further explained that students must evaluate situations, select problem-solving strategies, draw logical conclusions, develop, describe, and reflect on solutions when engaging in the mathematical reasoning process. Therefore, mathematical reasoning habits can be developed after students engage in plenty of learning experiences using numbers, quantities, numerical relationships, and problem-solving (Oslington et al., 2020).

Current mathematics curricula emphasize the reasoning process as one of the key mathematical practices. Consequently, the teacher should prepare an effective environment for nurturing mathematical reasoning by deliberately choosing tasks and activities that require students to engage in mathematical reasoning. In short, the teacher's role when it comes to providing opportunities to practice the reasoning habit is significant (Tall, 2014). However, Thai students are relatively self-conscious, and their natural inquisitiveness is not expressed as much as possible. It is perhaps caused by unfortunate approaches used in teaching mathematics, which prevent the students from being curious, asking questions, and engaging in reasoning. It means that students are unable to connect ideas, gain a deeper conceptual understanding, and ultimately enjoy learning mathematics (Thinwiangthong et al., 2020). In fact, in the current context of mathematics classes in Thailand, teachers still fail to utilize cognitive factors to promote students' mathematical reasoning habits in terms of mathematical and learning processes. The non-functional status quo offers an essential opportunity for educators to implement Lesson Study (LS) and the Open Approach (OA) to support the development of the cognitive aspects of mathematical reasoning. Students' habits, such as an effort, would contribute useful educational insights for teachers in formulations of meaningful teaching.

Over recent decades, multiple studies have provided plentiful evidence of the value of providing opportunities for students to engage in normal statistical reasoning from the earliest years of schooling (Aridor 
\& Ben-Zvi, 2017; Doerr et al., 2017; English, 2013; Lehrer \& English, 2018; Makar, 2016; Makar \& Rubin, 2017). For example, English (2013) argued that elementary school students could make informal inferences in uncertain situations to ensure a solid learning foundation for formal statistical understanding in later years. Moreover, Makar and Rubin (2017) asked students to interpret data from familiar sources. As a result, they were exposed to some big ideas in statistics, such as variability, range, and the aggregate properties of data sets. They found that multiple alternatives are often reasonable. Hence, teachers have to understand students' mathematical thinking (Stigler \& Hiebert, 1999), while students have to improve their mathematical reasoning habits in order to develop the relevant ideas (Cuoco et al., 1996) and use them to enhance their mathematical competence (Kilpatrick et al., 2001). Even though mathematical reasoning habits are recognized as important components of mathematical learning, students are not adequately trained to adopt them. Consequently, many students are reported to be facing difficulties to possess them (Foster, 2012). One of the significant barriers was that teachers liked to emphasize the memorizing approach and did not encourage students to engage in autonomous learning to cultivate mathematical reasoning habits (Wagner, 2008; Zhao, 2009). This was later supported by Inprasitha (2014). Inprasitha raised the problems that teachers tend to use rote memorization to teach mathematics, instead of using their creativity to enhance the students' problemsolving skills. It indicates that creative and innovative teaching is incredibly important to enhance mathematical reasoning habits.

\section{CONCEPTUAL FRAMEWORK}

A long-established practice of lesson preparation that originated in Japan, the socalled lesson study (LS) approach, has been in existence for over 130 years (Tall, 2008). LS is defined as a teacher-led instructional improvement cycle in which teachers work together to convey student learning targets, design a lesson, teach and/or observe the lesson, reflect on the collected evidence, go through the lesson for improvement, and re-teach the reviewed lesson (Lewis, 2002; Takahashi \& Yoshida, 2004). Moreover, LS is considered a professional learning model based on collaboration, which supports mathematics teachers' learning about content, curriculum, and student thinking, and facilitates greater reflection and more focus on conversations than traditional types of professional development (Hart et al., 2011). LS has been implemented to improve lessons in actual classroom contexts, and it is considered one of the most effective strategies to enhance teaching, particularly for mathematical subjects (Loipha \& Inprasitha, 2004). Because of LS's versatility, innovation has continued to be a vital component for teacher development and has become well known and popular in the teaching profession (Baba, 2007). LS was first introduced and adapted to the Thai 
educational context in 2002, encompassing three steps: (i) design a research lesson collaboratively (Plan); (ii) observe the research lesson collaboratively (Do), and (iii) discuss and reflect on the research lesson (See) collaboratively (Inprasitha, 2009).

The open approach (OA) also originated in Japan. The ideas underpinning OA are targeted at permitting students to learn mathematics in response to their mathematical ability, complemented by a certain degree of self-determination in their learning. This latter can improve the quality of the learning processes and result in mathematics achievement (Nohda, 2000). Later, Inprasitha (2011) proposed an OA that consisted of four phases, namely (i) posing open-ended problems; (ii) encouraging students' self-learning; (iii) introducing whole-class discussion and comparison, and (iv) summarize by connecting students' mathematical ideas that have emerged in the classroom by incorporating $\mathrm{OA}$ into the three-step LS model. The researchers adopted Inprasitha's $(2011,2014)$ conceptual framework as elucidated in Figure 1 in this study. Moreover, Thinwiangthong et al. (2020) found that the LS and OA framework can encourage mathematics teachers to foster a positive learning environment with regard to any learning activities. According to Loipha and Inprasitha (2004), integrating the OA and LS process involves encouraging students to access alternative perspectives, methods, and answers through dealing with open-ended problems, which could enhance students' reasoning skills.
According to Isoda (2015), two types of theories underpin the incorporation of $\mathrm{OA}$ into the LS process, namely problem-solving approach and theories for curriculum. Shimizu (1999) explained the problemsolving approach as a well-known Japanese teaching method for understanding and turned it up as a shared teaching theory to develop students to learn mathematics by and for themselves. On the other hand, Isoda and Katagiri (2012) described the problem-solving approach as teaching about learning how to learn and teaching values so that students are being able to use their mathematical thinking in solving problems as one of the most fundamental goals of teaching mathematics. Theories for the curriculum are another theory used, as illustrated in teachers' guidebooks in the form of several technical terms, which are only used by mathematics teachers and educators to share mathematical knowledge for teaching (Isoda, 2015).

The National Council of Teachers of Mathematics (NCTM, 2009) builds on the ideas of NCTM's Principles and Standards for School Mathematics and focuses on how school mathematics can better prepare students for future success through mathematical reasoning as the heart of school curriculum. By following the proposal of the NCTM (2009) with regard to the conceptual framework, the researchers have conceptualized the cognitive aspects of mathematical reasoning habits in four steps as follows: (i) analyzing a problem; (ii) implementing a strategy; (iii) 
using connections, and (iv) reflecting on a solution. The NCTM (2009) recognized mathematical reasoning habits as productive ways of thinking in mathematical inquiries and sense-making. The rationale of fostering student reasoning habits is addressing the direction of school mathematics in the $21^{\text {st }}$ century because it involves three critical aspects: (i) meta-cognition (reflecting on and identifying one's thinking processes), (ii) the role of student discourse in promoting student reasoning, and (iii) the importance of providing equitable opportunities for all students to engage in and share their reasoning. The three critical aspects correspond to the main ideas of OA and LS, as students need to explain their thinking after they have had their self-learning and discussion with their peers.

In the 'Plan' step of LS, the LS group is teachers discussing the relevant contents and teaching activities required for students to utilize their cognitive aspects of mathematical reasoning habits in the OA problem-solving process. This step mainly highlights how mathematical reasoning acts as the 'glue' that helps mathematics make sense. Finally, data will be collected through the 'Do' step of LS. In other words, the researchers will determine all the cognitive aspects of mathematical reasoning habits through their observations, interviews, and consideration of the students' worksheets, to refine their understanding of what students mean by the term 'mathematical reasoning.'
Martin and Kasmer (2010) mentioned that mathematical reasoning habits should be integrated into and implemented with various curricular topics in all mathematics classes and at all school levels. These aspects are essential in mathematics learning as they ensure that students are equipped with the necessary understanding and the ability to apply such understanding in practice. On the other hand, Cuoco et al. (1996) studied the relevance of mathematical reasoning habits in mathematical learning and found that the mechanisms with regard to promoting students' understanding are still not given sufficient attention. Teachers primarily attempt to prepare students for tests to meet designated standards (NCTM, 2000). Occasionally, teachers have immediately revealed answers and guided them straight away. This phenomenon reflects that students rarely have adequate time to develop their mathematical reasoning habits (Foster, 2012).

In this context, the researchers would like to explore how the cognitive aspects of students' mathematical reasoning habits relate to integrating LS and OA into the mathematics subject teaching activities of $3^{\text {rd }}$ Grade elementary school students. In short, LS is a tool to improve the teaching approach, while OA is a teaching approach and an aspect of LS (Inprasitha, 2011). 


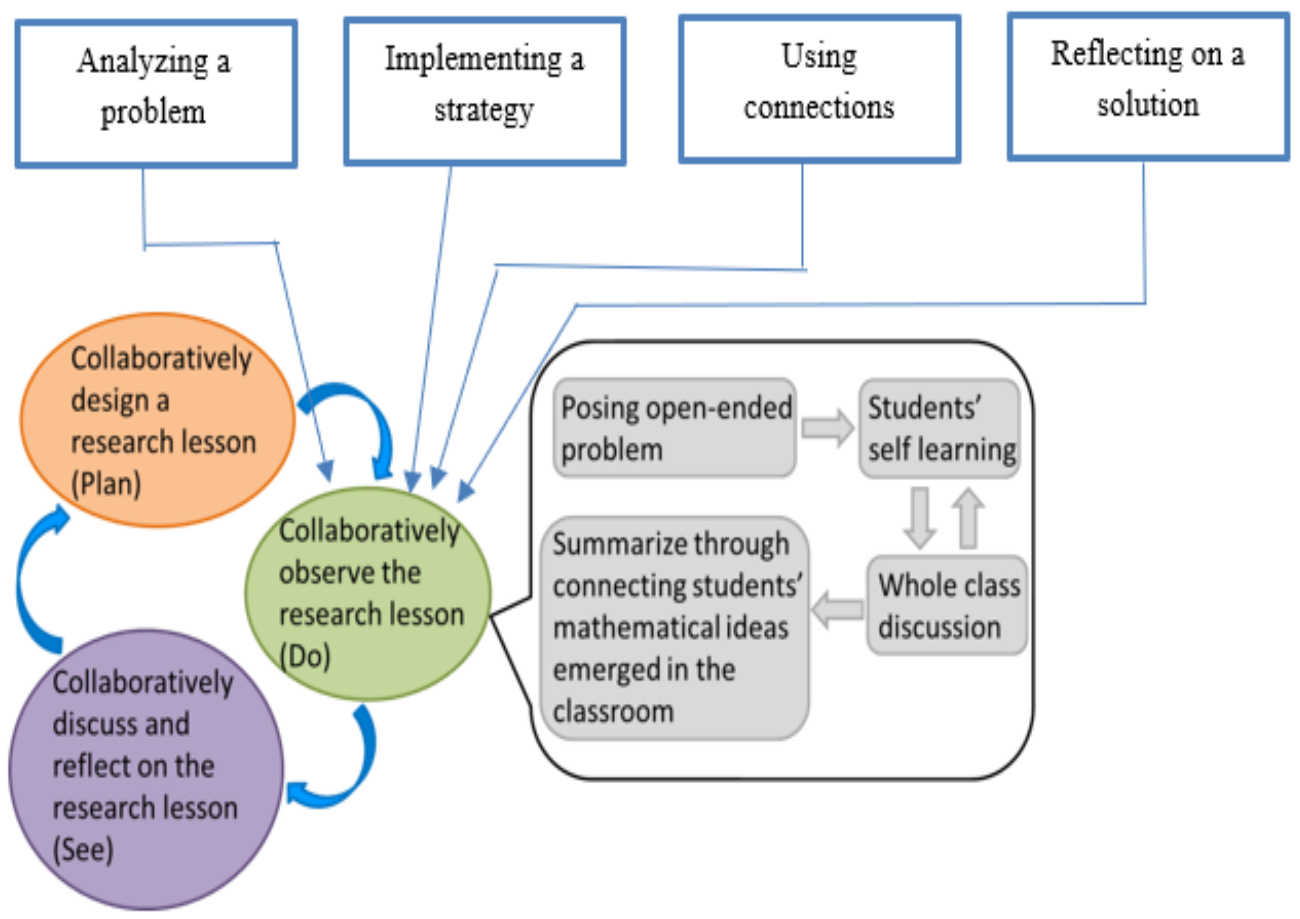

Figure 1. Conceptual framework

\section{METHODS}

\section{Study Samples}

The target group comprised eight $3^{\text {rd }}$ grade students (Elementary $3 / 2$ ) during Semester 2 the academic year 2018 from Ban Nonsankham Nongsala Sisa-at School, Sisaket Province, Thailand. These eight individuals were purposively selected because they had participated in a teacher professional development project for mathematics financially supported by the Education Equity Fund and conducted by the Centre for Research in Mathematics Education, Faculty of Education, Khon Kaen University, Thailand. The whole school approach was applied to the research school by incorporating OA into the LS process in every mathematics lesson for 96 LS cycles over the past two years (2016 to 2018). It implies a collective and collaborative action in and by a school community to improve student learning and the conditions that support the OA incorporated into the LS process as the teaching innovations.

The purposive sampling technique was appropriate in this study involving consideration of students' behaviors and work outcomes, aimed at demonstrating the cognitive aspects of mathematical reasoning habits. In addition, a teacher advisor was interviewed to identify the characteristics of each student. After that, researchers used tracking practice to create four homogeneous groups of students, with 
the assumption that this would make it easier for teachers to tailor instruction to students' needs. Finally, the eight samples were identified using cross-case analysis.

\section{Study Design}

Researchers employed a qualitative approach aiming to convey meaning and comprehension via a detailed description. In this sense, the qualitative approach is a powerful method for exploring educational issues in that it allows us to develop a comprehensive view of complex social settings. Therefore, the qualitative approach is suitable for identifying the mathematical reasoning habits of the samples' real-life experiences within those settings. A crosscase study research design was employed as it offers the opportunity to blend several methods, which justify the study approach. The cross-case analysis was built upon the mathematical reasoning habits found in the specific cases. Data for this qualitative approach were obtained from several different sources in interviews, observations of real-life settings, and documents.

\section{Study Procedure, Instruments, and Data Collection}

Students' mathematical reasoning habits were hypothesized to improve after the OA treatment in the LS process was used for teaching mathematics. The research procedure involved three phases. An LS team was formed and worked collaboratively to develop lesson plans for the $3^{\text {rd }}$ Grade mathematics topic 'Division with Remainders' by incorporating OA in the first phase. The second phase followed it. All LS team members observed the teaching by focusing on the cognitive aspects of the mathematical reasoning habits of the students except the teacher who was teaching. In the final phase, the LS team members discussed and reflected on the teaching results, examined the observationderived results, and attempted to improve the research lesson.

The learning observation was constructed using the framework of NCTM (2009) regarding four cognitive aspects as follows: (i) analyzing a problem; (ii) implementing a strategy; (iii) using connections; and (iv) reflecting on a solution. Two instruments were used, namely students' worksheets and field notes, along with the learning observation. Students' worksheets were in the form of written assignments, including the records of students who used mathematical ideas to solve mathematical problems, who offered problem solutions involving logical explanations. In addition, students' worksheets and field notes were used to record all the problem-solving behaviors and cognitive aspects of mathematical reasoning through the lesson observation.

In the final phase, semi-structured interviews were conducted with the eight participants to collect qualitative data. The interview protocol was designed mainly to explore the eight participants' views regarding how they analyzed the problem, how they implemented strategies in solving the mathematical problems, how they made connections to solve the problems, 
and how they reflected on the solution. The students' logical thinking to produce ideas was our major interest. Therefore, the researchers had to ensure that the interview questions were aligned with the research questions before engaging in an inquirybased conversation. The research questions required students to explain their thinking and how they believe their solutions make sense. Data were analyzed using content analysis.

\section{RESULTS}

The results of this study are presented by following the aims indicated above. The three steps of the LS framework (Inprasitha, 2011) were employed as a study procedure, while the OA (Inprasitha, 2011) was operated as a treatment during the second step of the LS framework. Finally, the researchers used the NCTM (2009) framework to identify the four cognitive aspects of mathematical reasoning habits.

\section{Step 1 of LS: Collaboratively Design a Research Lesson (Plan)}

The study was begun by creating an LS team consisting of a mentor, researcher, and two mathematics teachers to design a lesson plan. When designing the lesson plan, the LS team had to fit in the overall development of the particular topic content that was 'Division with Remainders' as part of the $3^{\text {rd }}$ Grade mathematical syllabus. They also must stipulate the main objectives of the lessons and the comprehensive development of the lesson arrangement by taking into account the OA treatment. In other words, the LS team organized the lesson plans in sequence so that an appropriate range of mathematical reasoning habits was taken into consideration (Askew, 2020). In this way, the possible solutions were planned so that the teacher could organize a coherent discussion based upon the following problem situation (Tall, 2014). Figure 2 shows the LS team creating a joint lesson plan.

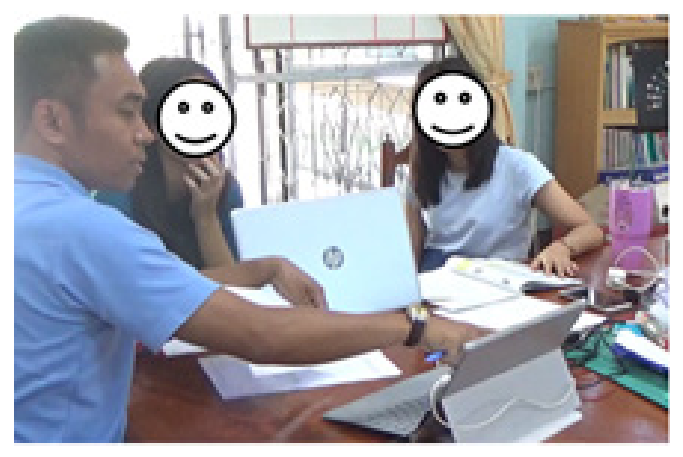

Figure 2. LS team was creating a joint lesson plan

The problem situation was derived from the topic "Division with Remainders" in the $3^{\text {rd }}$ Grade mathematics textbook (Inprasitha, 2010, p. 45), as shown in Figure 3:

"If you have 20 apples and 23 oranges, how many bags do you need to fit four of each kind of fruit in each bag?"

Instruction: Write a corresponding mathematical statement and provide an answer. 

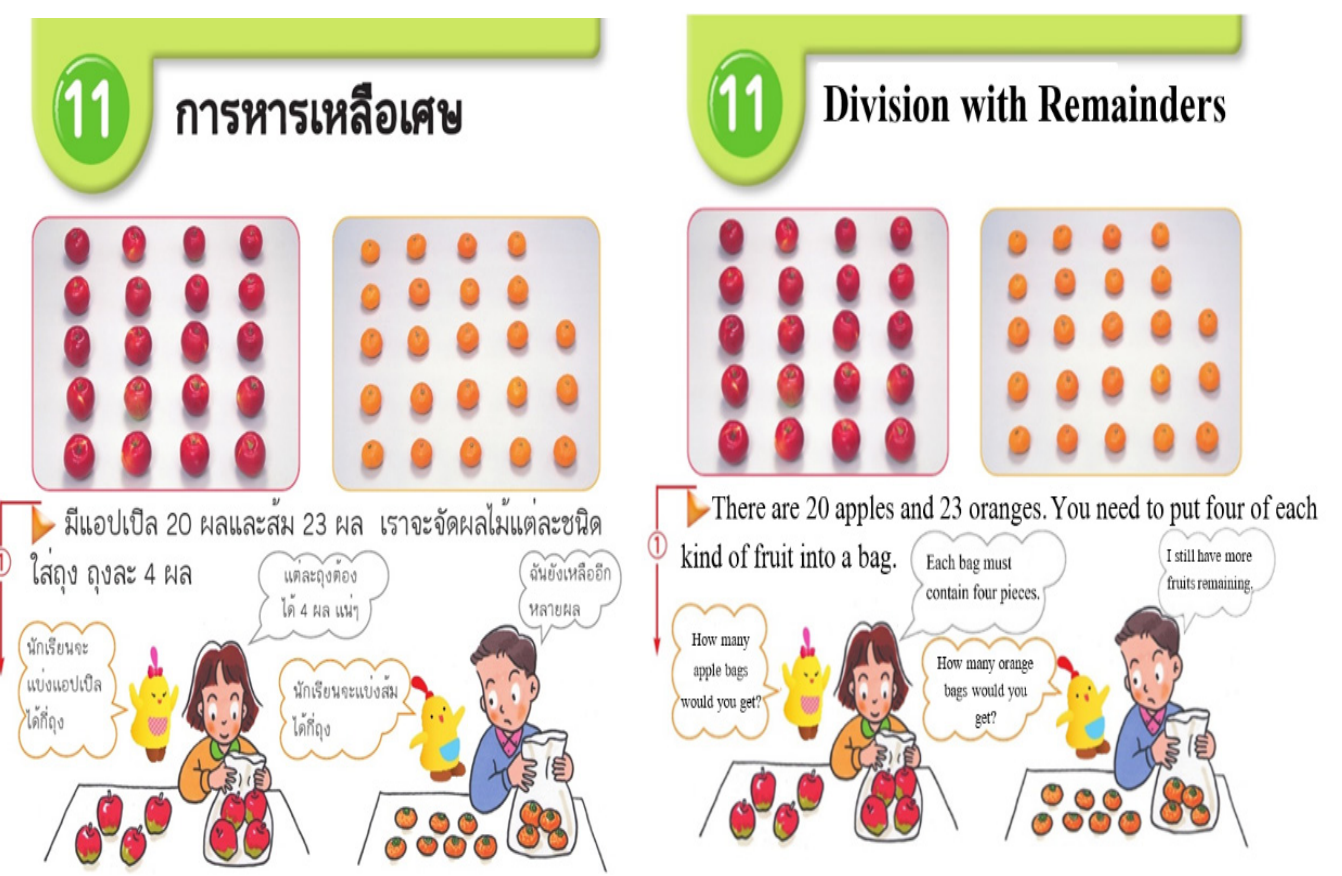

Figure 3. Problem situation

\section{Step 2 of LS: Collaboratively Observe the Research Lesson (Do)}

As indicated in Step 1 of the LS procedure, the LS team members planned the research lesson collaboratively. It was followed by Step 2, whereby the research lesson was conducted by a mathematics teacher (an LS team member), with a group of supportive observers (the other LS team members) who discussed and reflected on the research lesson immediately after the lesson had finished. In this step, it is important to have a group of observers (the LS team members) who have ideas fresh in their minds because they can then have a comprehensive focus on every cognitive aspect of the mathematical reasoning habits.

The observers stayed at the back or sides of the classroom as the lesson proceeded, but when the students were working on the problem, they were able to walk around and observe how students approached the problem differently. At this point, they could make field notes, ask the students why they had solved the problems in such a way, and so on. The main intention was to obtain a coherent overall view of the research lesson in terms of the students' mathematical reasoning habits. Figure 4 elucidates Step 2 of the LS approach. 


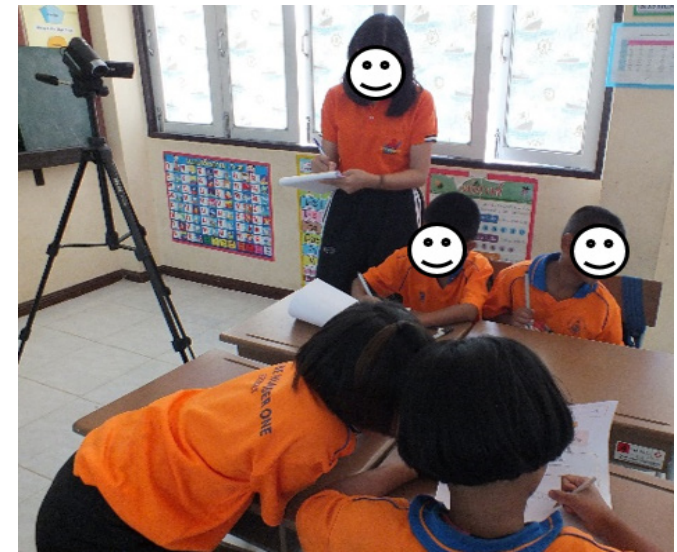

Figure 4. Step 2 of LS implementation

As part of the second step of the LS approach, the teacher integrated the four OA steps into their teaching practices according to the research lesson plan as follows:

(i) Posing an open-ended problem

The teacher-reviewed the students' prior knowledge before starting her teaching activities. Therefore, she asked her students what they had learned previously. This was followed by posing the problem situation by teacher with the instruction on the chalkboard. The problem situation was "If there were 20 apples and 23 oranges, how many bags would be needed if you have to fit four of each kind of fruit in each bag?". She then requested the students to read the problem situation and its instruction together. After that, she identified students' understanding of the problem situation, as shown in Figure 5.

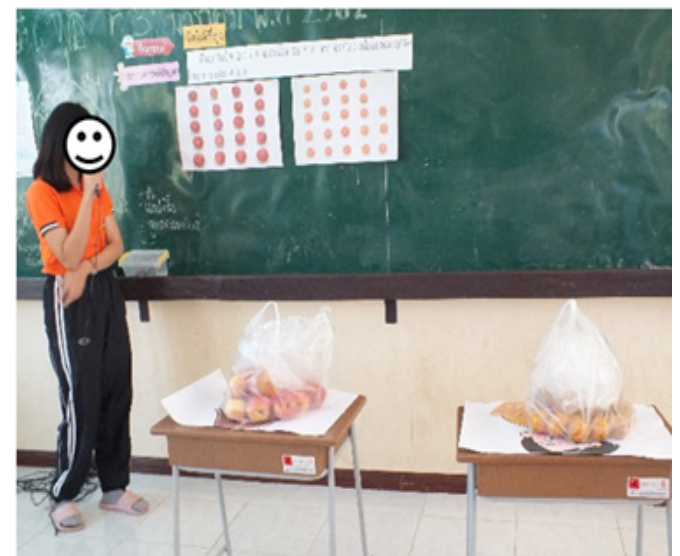

Figure 5. Posing open-ended problem

(ii) Students' self-learning

In the second step of OA, the students were required to read the problem situation and the associated instruction and were encouraged to discuss the problem situation with their peers. Subsequently, the teacher distributed the worksheets and teaching aid to each group of students to solve the problem together, as shown in Figure 6.

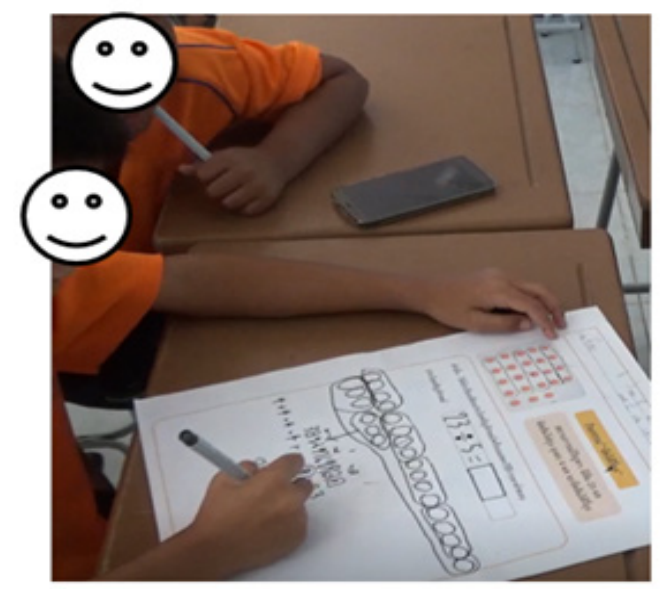

Figure 6. Students' self-learning to solve the problem 
(iii) Whole class discussion and comparison After the students had finished solving the problem and submitted their worksheets to the teacher, the teacher selected the groups with different mathematical reasoning habits to present their views about how they solved the problem. At this step, students were encouraged to learn collaboratively, and the teacher gave sufficient opportunities for their peers to give their viewpoints. Figure 7 shows the activity of whole-class discussion and makes a comparison.

After the students had finished solving the problem and had submitted their worksheets to the teacher, the teacher selected groups with different mathematical reasoning habits to present their views about how they had solved the problem. At this stage, the students were encouraged to learn collaboratively, and the teacher gave sufficient opportunities for their peers to give their viewpoints. Figure 7 shows the activity of whole-class discussion, and the teacher made a comparison.

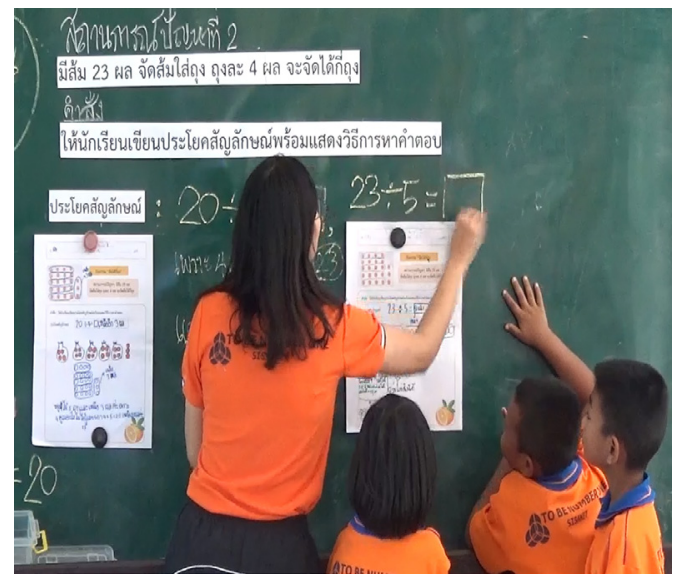

Figure 7. Whole class discussion and comparison (iv) Summarize by merging students' mathematical ideas in the classroom.

After the students had completed their group work presentation, the teacher posted their worksheets on the chalkboard. The students were then required to explain their mathematical reasoning verbally, and the teacher wrote down all the additional information on the worksheets. Finally, the teacher summarized the lesson by compiling all the students' viewpoints according to the worksheets on the chalkboard. Then, all the students were allowed to share their viewpoints, as shown in Figure 8.

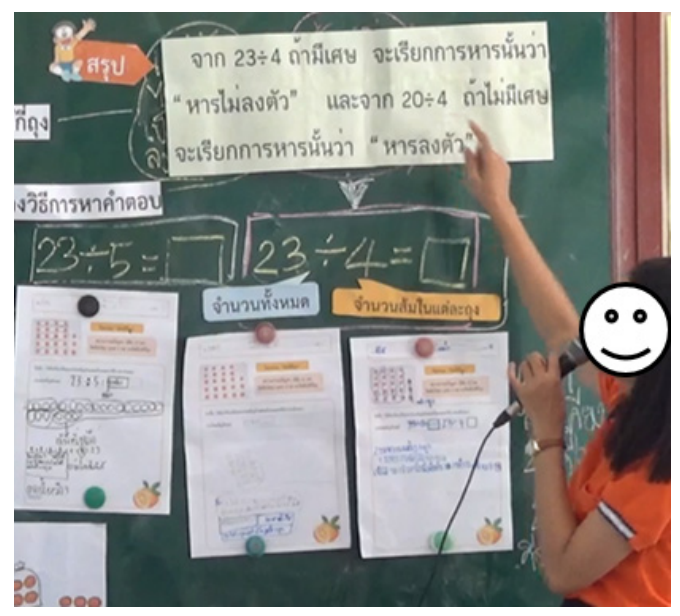

Figure 8. Summarizing by connecting students' mathematical reasoning habits

\section{Step 3 of LS: Collaboratively Discuss and Reflect on the Research Lesson (See)}

During Step 2 of LS, the members of the LS team observed and collected the data with regard to the students' mathematical reasoning habits. A reflection session 
was conducted after the research lesson mainly dealing with the learning activities based on the collected data. According to Tan et al. (2017), there are three main advantages of Step 3: (i) As the observing teachers observe another teacher's lesson, they can share their views to create a clearer or more comprehensive picture of mathematical reasoning habits as demonstrated by students; (ii) the teacher who teaches the research lesson can realize their strengths and weaknesses from the observing teachers' feedback, and (iii) the observing teachers learn as they observe the teaching of another teacher. In sum, Step 3 of the LS approach is important because the LS team members were able to discuss and reflect on the research lesson collaboratively, allowing them to identify the pieces of evidence relating to the students' mathematical reasoning habits. Thus, this process can promote the mathematics teachers' professionalism. Figure 9 elucidates Step 3 of LS.

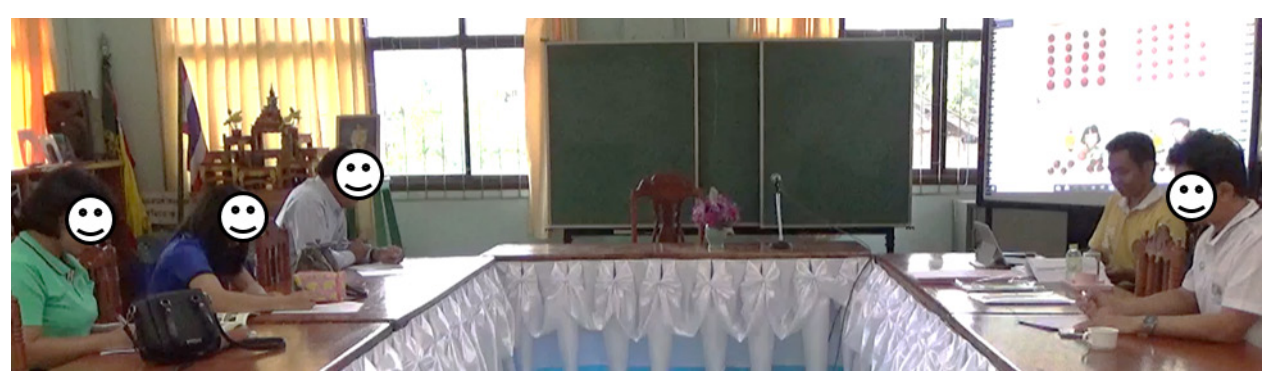

Figure 9. LS team members discussed and reflected on the research lesson

\section{Study Outcomes: The Four Cognitive Aspects of Mathematical Reasoning Habits}

The outcomes of this study are presented by following the framework of NCTM (2009) with regard to the four cognitive aspects of mathematical reasoning habits. The eight specific cases were identified and labeled as $\mathrm{S} 1$ to $\mathrm{S} 8$.

(i) Cognitive aspect 1: Analyzing a problem

In this step, a protocol analysis was conducted to examine the cognitive aspect described as "Analyzing a problem" using the problem situation detailed on the activity sheet. The data was obtained from a video recording of the class activities during the research lesson. The researchers transcribed the records, identified behaviors in textual form, and created a protocol showing the verbal expressions and behaviors of the students. It is elaborated as follows:

Item 1 S1: Write here.

Item $2 \mathrm{~S} 2$ and S3: There are 23 oranges; by putting four into a bag, how many bags of oranges would be there?

Instructions: Write a corresponding mathematical statement and provide an answer. 

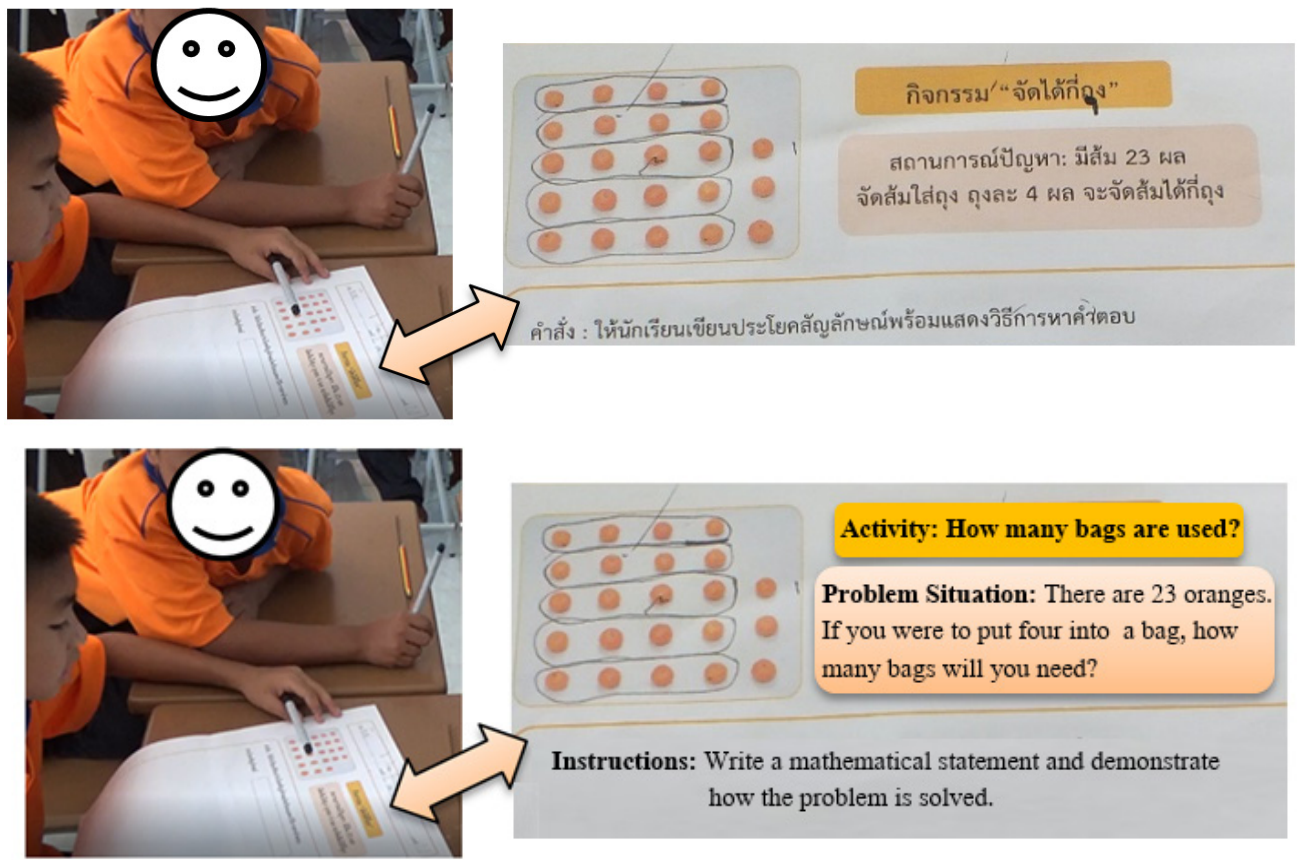

Problem Situation: There are 23 oranges. If you were to put four into a bag, how many bags will you need?

Instructions: Write a mathematical statement and demonstrate how the problem is solved.

Figure 10. The students are analyzing a problem from the worksheet

The qualitative result obtained from the verbal expressions and behaviors of the the field notes and the interviews revealed students as elaborated below:

\section{1. นักเรียนวิเคราะห์ปัญหาอย่างไร}

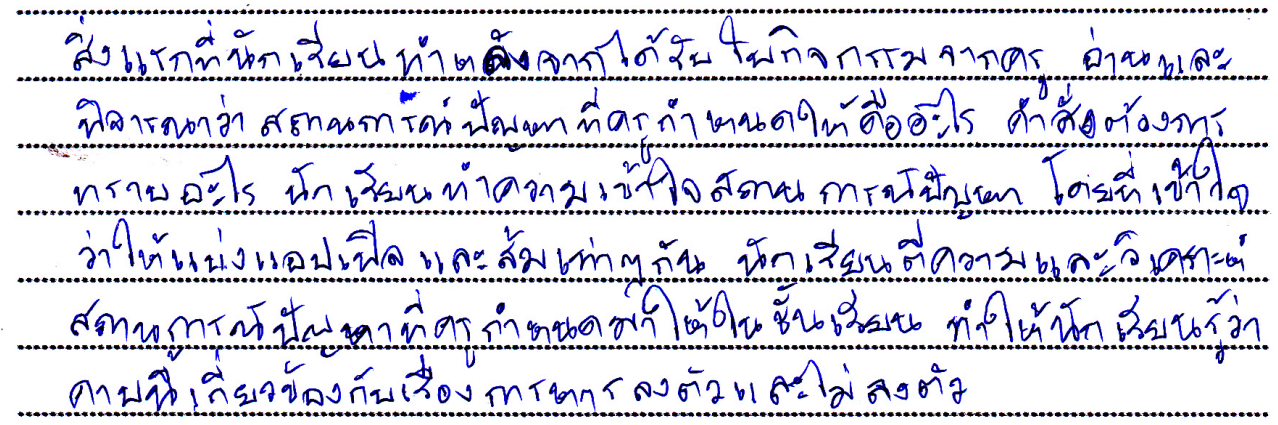

Figure 11. An excerpt from the field notes demonstrating how the students analyzed a problem 


\section{Translation}

Upon receiving the activity sheet from the teacher, the first action the students took was to read and determine what the problem situation required and what the instructions expected. The students attempted to understand the situation. They understood that apples and oranges must be equally distributed. They interpreted and analyzed the assigned problem situation in class and found out that the period was going to be about divisions with and without remainders.

Figure 11 (Continue)

As exhibited in Figure 11 (field notes) above, the statement "Students interpreted and analyzed the problem situation assigned by the teacher in the classroom" was evidence to show that the students jointly analyzed the problem until they began to realize that they were going to study divisions with remainders.

Interviewer: What are you doing here?

Item 2 S4: I am reading the instructions.

Item 3 Interviewer: What did you do while analyzing the problem?

Item 4 S5: We read, discussed, and tried to understand it together. So, we were able to do it.

Figure 12. Interview results

The above results revealed that the students helped each other (Figure 10) to understand and interpret the problem situation (Figure 11) and that the students read the problem situation together (Figure 12). The other evidence emerged from the interview analysis from Item 4, when Student 5 (Figure 12) stated, "We read, discussed, and tried to understand it together. So, we were able to do it." This verbatim statement reflects that the students were aware of their roles in mathematical learning while internalizing and understanding the problem related to division with and without remainders. When the researchers triangulated data from various sources, namely photos, field notes, and semi- structured interviews, the qualitative results revealed that this was the cognitive aspect of the mathematical reasoning habits and could be categorized as the 'analyzing a problem' aspect.

(ii) Cognitive aspect 2: Implementing a strategy

The second aspect relating to mathematical reasoning habits was conducted to examine the students' cognitive aspects regarding implementing a strategy by using multiplication to solve the problem situation on their activity worksheets. Figure 13 shows the cognitive aspect of the students' mathematical reasoning habits through the way they implemented a strategy to solve the problem situation detailed in their 
activity worksheets. It is identified as the first piece of evidence of the second aspect of their mathematical reasoning habits.

Item 3 S6: Four, five, twenty

Item 4 S7: How many bags are needed for the distribution? There is no mathematical statement for it.

$$
\begin{gathered}
4 \times 1=4, \quad 4 \times 2=8, \quad 4 \times 4=16, \\
4 \times 4=16,4 \times 5=20,4 \times 6=24
\end{gathered}
$$

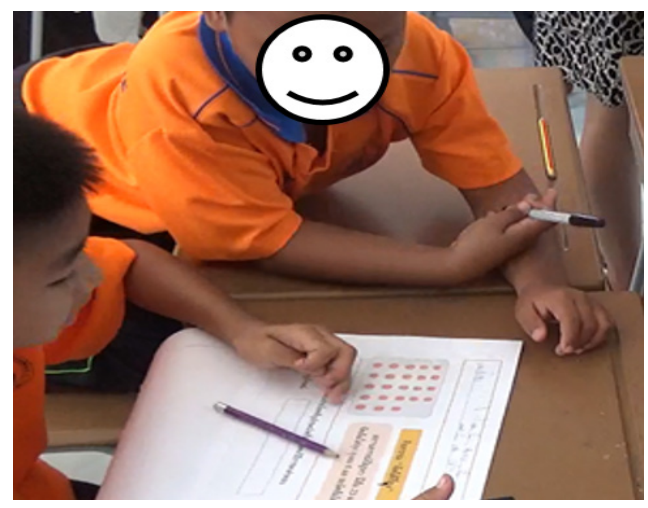

Figure 13. Implementing a strategy through multiplication to solve a problem
The second piece of evidence was obtained from the field notes ad indicated that the students were implementing a strategy while studying mathematics during the research lesson. Figure 14 shows the results from the field notes.

The third piece of evidence was obtained from the semi-structured interviews, as shown in Figure 15.

The three pieces of evidence were then triangulated and were concluded as the second aspect of mathematical reasoning habits, namely implementing a strategy as follows: The first evidence from the students' worksheets indicated that Table Four of the multiplication formula was utilized as elucidated in Figure 13. Figure 13 shows that the students applied a mathematical idea known as Table Four of the multiplication formula as their strategy to solve the problem. The second piece of evidence was derived from the field notes.

\section{2. นักเรียนใช้ขุทธวิธีในการแก้ปัญหาอย่างไร (วิธีคิด หรือวิธีการแก้ปัญหา)}

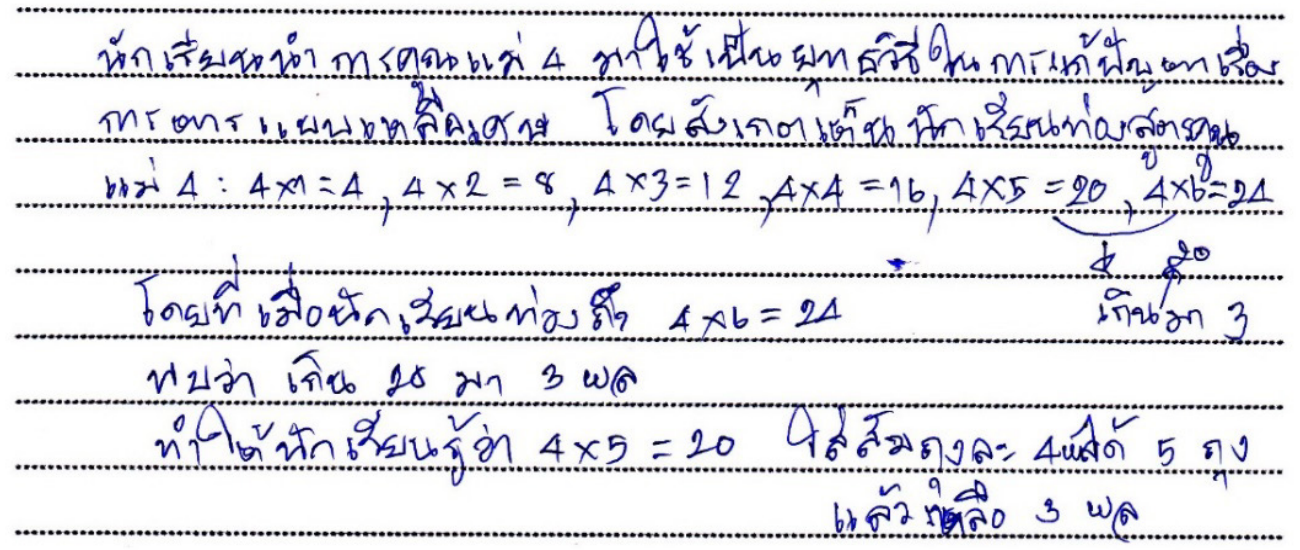

Figure 14. Field notes (Second aspect of implementing a strategy) 


\section{How did the students implement a strategy in problem-solving? \\ The students used Table Four of the multiplication formula as the strategy for solving the division with remainders. It was observable that they recited Table Four: $4 \times 1=4,4 \times 2=8$, $4 \times 3=12,4 \times 4=16,4 \times 5=20$, and $4 \times 6=24$. So, 3 exceeded 20 . Once they reached $4 \times 6=24$, they discovered that there were three extra fruits beyond 20 . Hence, they understood that $4 \times 5=20$ means the oranges could be put into five bags of four each with three as the remainder.}

Figure 14. (Continue)

Item 15 Interviewer: What was your teacher's question that led you to use this solution to solve the problem?

Item 16 S8: Multiplication: Four multiplied by five, twenty, and the remainder is three.

Item 17 S1: Student 8 spoke, and the teacher wrote.

Item 18 Interviewer: Yes! The teacher wrote after Student 8 had spoken.

Figure 15. Semi-structured interviews with students (Second aspect of implementing a strategy)

The identified statement was, "The students used Table Four of the multiplication formula as the strategy to solve the division with remainders." This evidence was identified while the students scaled the Table Four formula until they reached $4 \times 5=20$. The problem was that since there were 23 oranges in total, the students realized that three were left as a reminder following the division. Hence, multiplication was used as a strategy to solve the division problem with remainders. The final evidence emerged from the semi-structured interviews (Figure 15), Item 16, Student 8's statement as "Multiplication: Four multiplied by five, twenty, and the remainder is three". This statement indicated the use of multiplication as a strategy to solve a division problem with remainders, and that the students realized that $4 \times 5=20$ and the remainder was 3 . The researchers then triangulated, concluded, and categorized the three pieces of evidence as to the cognitive aspect of mathematical reasoning habits as 'implementing a strategy' aspect.

(iii) Cognitive aspect 3: Using connections The third aspect of mathematical reasoning habits was considered by examining the cognitive aspects related to using connections to solve the problem situation on the students' activity worksheets. Figure 16 shows the cognitive aspect of mathematical reasoning habits through the way the students use connections to solve the problem situation detailed in their activity worksheets. This is identified as the first evidence of the third aspect of mathematical reasoning habits, as shown in Figure 16.

Item 5 S3: It won't work.

Item 6 S4: Why not circle four at a time? Just circle them. 

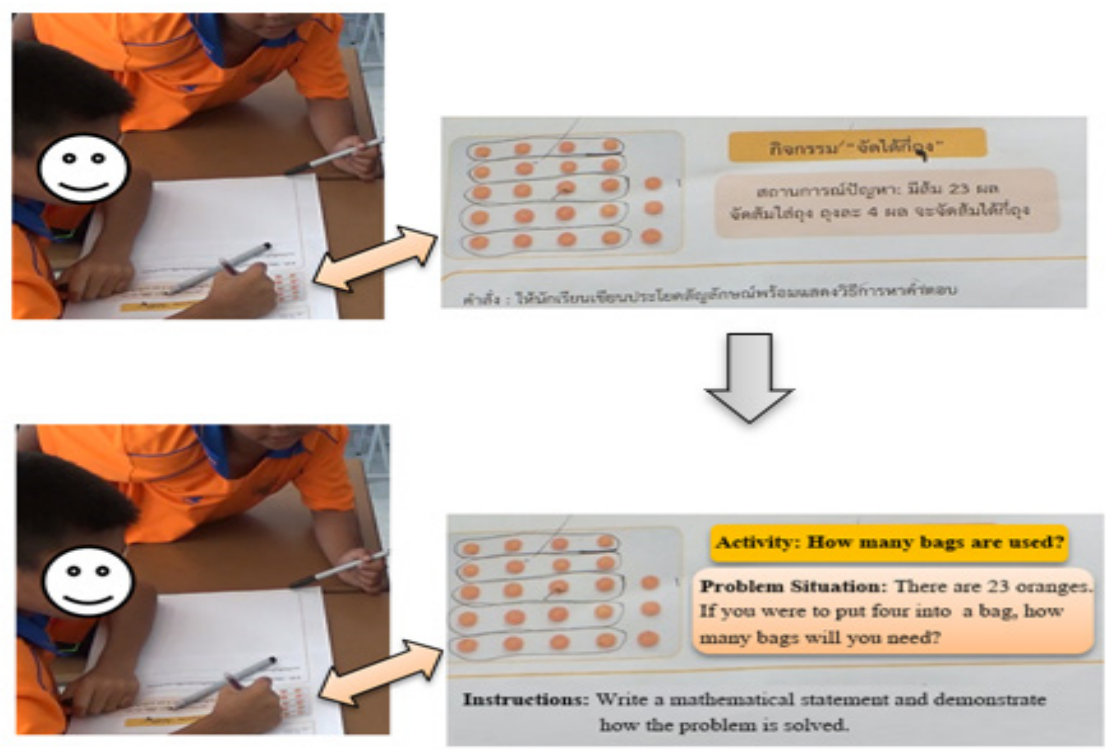

Figure 16. Students' writings indicate an association of the image-circling idea to solve problem

3. นักเรียนค้นหาและเชื่อมโยงการแก้ปัญหาอย่างไร

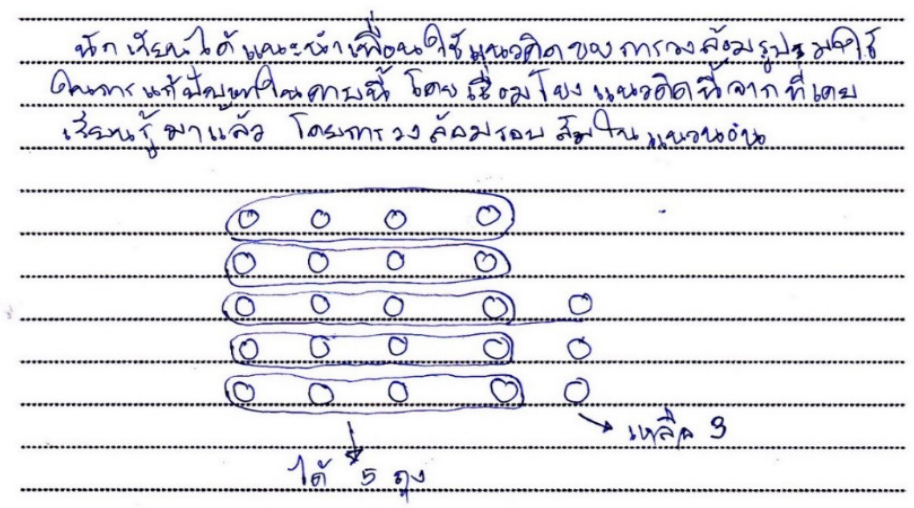

3. How did the students use connections in problem-solving?

A student suggested to his peers that the idea of image circling could be used to solve the problem under consideration. The connection was made by referring to an idea which is based on previous knowledge, and the students drew horizontal ovals for the oranges. This could relate to multiplication by grouping.

Figure 17. Field notes that demonstrate how students used connections 
The third piece of evidence was obtained shown in Figure 18. from the semi-structured interview results as

Item 22 Interviewer: Where did you learn about the circling technique that you are using? Item 23 S5: Elementary 2

Item 24 Interviewer: Are you learning about the circling technique during Elementary 2?

Item 25, S5: Yes, I was using the circling technique that I learned in Elementary 2 to solve most of the mathematical problems.

Item 26 Interviewer: So, it is using the knowledge that you are getting in Elementary 2, correct?

Figure 18. Semi-structured interviews with students (the third aspect of using connections)

The above three pieces of evidence were then triangulated and concluded as the third aspect of mathematical reasoning habits, namely using connections. So, it goes as follows: The first evidence is derived from Item 5, when Student 3 said, "It won't work." This signaled that a new method was required. Afterwards, Student 4 came up with a previously learned idea that involves circling four oranges at a time and suggested to Student 3 that he connect this idea with problem-solving and write the solution onto the activity worksheet. Again, in Item 6: "Why not circle four at a time? Just circle them". Moreover, a photo of the students establishing a connection through circling is shown in Figure 16 and is further confirmed as the first piece of evidence. Next, the second piece of evidence was from the field notes, specifically in the text that states, "A student suggested that his peers employ the circling strategy to solve the problem under consideration by connecting the previouslylearned idea to the problem-solving". During the time, the researchers noticed that the students made a connection by circling the oranges horizontally, forming five bags, with three being left as a remainder. This indicates how the students connected the circling idea with "Division with Remainder" (Figure 17). The final piece of evidence emerged from the semi-structured interviews with the students 5 through the statement: "Yes, I am using the circling knowledge that I learned in Elementary 2 to solve the problem. " This verbatim statement was logged when the students connected the image circling knowledge from Elementary 2 to the problem-solving for 'Division with Remainder'. Finally, the researchers triangulated, concluded, and categorized the three pieces of evidence as to the cognitive aspect of mathematical reasoning habits, namely the 'using connections' aspect.

(iv) Cognitive aspect 4: Reflecting on a solution

The final aspect of mathematical reasoning habits was carried out to examine the students' cognitive aspect of reflecting on a solution onto their activity worksheets as follows: 
Item 7 Student 1: $4+4+4+4+4+3$ the answer simultaneously). $=23$ (The student told his peers and wrote

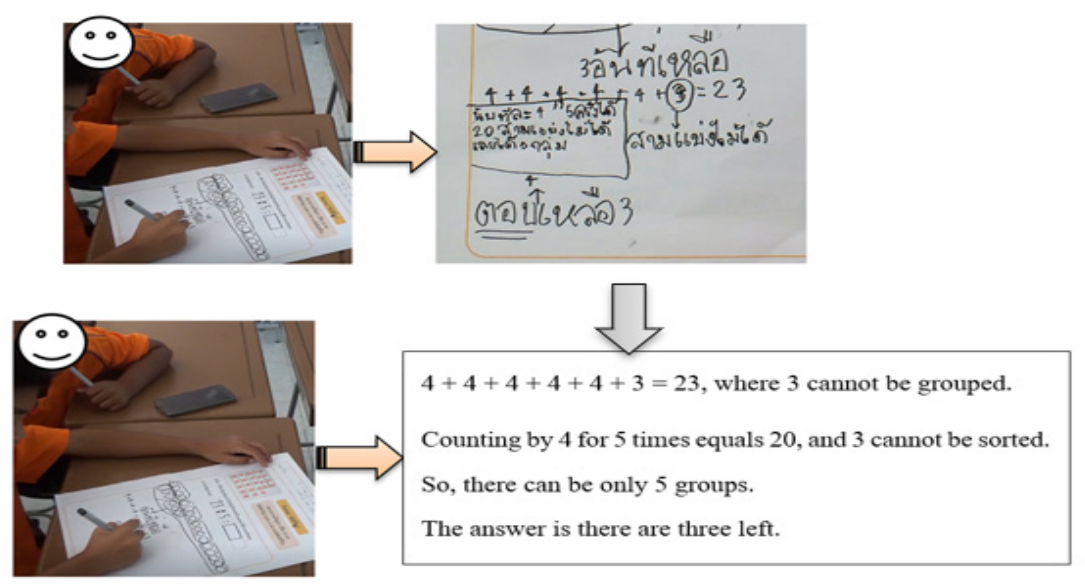

Figure 19. Students' writings indicate their reflection on a solution

The second piece of evidence that recorded during the research lesson. Thus, was obtained from the field notes, which the second piece of evidence relating to indicated that the students were reflecting the final aspect of mathematical reasoning on a solution, as their behavioral data were habits was elucidated in Figure 20.

\section{How did the students reflect on the solution?}

The students were observed to have implemented the addition strategy to validate and confirm the solving of the problem. After having studied, $4+4+4+4+4+3=23$, where number 3 was circled and indicated as non-groupable, they realized that the remainder was 3. The students counted by 4 and reached 20 oranges. However, the remaining 3 did not reach 4 , so it did not meet the condition.

Figure 20. Field notes indicating their reflection on a solution

The third piece of evidence was obtained shown in Figure 21.

from the semi-structured interview results as

Item 42 Interviewer: What did you use to confirm your answer?
Item 43 S6: I used addition.
Item 44 Interviewer: What was added?
Item 45 S6: Four
Item 46 Interviewer: How was it added?
Item 47 S7: $4+4+4+4+4+3=23$

Figure 21. Semi-structured interviews with students (the final aspect of reflecting on a solution) 
The above three pieces of evidence were then triangulated and were concluded as the final aspect of mathematical reasoning habits, namely reflecting on a solution. This went as follows: The first evidence was from the students' verbal explanation in Item 6, in which Student 1 spoke and wrote " $4+4+4+4+4+3=23$ " onto the activity worksheet. Moreover, a photo reflects that the students employed addition to confirm their problem-solving idea for division with remainders, specifically, by placing four oranges in bags at a time, and recognizing the remaining three as the remainder (Figure 19). This is followed by the second piece of evidence, which was derived from the field notes. The evidence statement is: "The students employed addition to review or validate the problemsolving”. This statement was recorded when the students were observed to be writing down the addition in this way: $4+4+4+$ $4+4+3=23$ and circled the number 3 as the remainder. In doing so they considered the conditions of the problem where four oranges were to be put into bags, making a total of five bags. Hence, they were able to produce a reasonable answer using addition to validate the image-circling idea to solve a division with the remainder (Figure 20). The final piece of evidence emerged from the semi-structured interviews with the students, as shown in Item 45, when Student 6 stated: "Four is added". The statement was mentioned when the students added four repeatedly, based on the number of bags of oranges, i.e., four per bag for a total of five bags with a three remainder. This was, as Student 7 stated in Item 47 , that " $4+4+4$
$+4+4+3=23$ ”. As the data from various sources were triangulated, it was found that this cognitive aspect of the mathematical reasoning habits could be categorized as "reflecting on a solution".

\section{DISCUSSION AND CONCLUSION}

The researchers explored the impact of OA treatment in the LS process on teaching practices in terms of enhancing the cognitive aspects of the mathematical reasoning habits of $3^{\text {rd }}$ Grade students. The results of this study clearly contribute to our recognition of the importance of OA treatment in the LS process while teaching mathematics, in terms of improving students' mathematical reasoning habits, such as (i) analyzing a problem; (ii) implementing a strategy; (iii) using connections, and (iv) reflecting on a solution in accordance with the framework of NCTM (2009). Therefore, this study extends the current literature by proposing a descriptive framework, not only for observing mathematical reasoning in elementary grade students, but also for mapping different developmental aspects of reasoning (Manmai et al., 2020). Furthermore, the results from various sources reveal that students can describe the mathematical concepts through their prior knowledge and adapting this to their problem-solving methods. This implies that students can find their own way regarding solving a problem, find various strategies, introduce different strategies to solve a problem, including being able to prove and interpret the outcomes through appropriate reasoning. 
Moreover, the results of this study correspond to Oslington et al.'s (2020) results. They found that mathematical reasoning habits can be developed after students engage in many learning experiences using number, quantity, numerical relationships, and problemsolving. In addition, our study procedure showed that the LS team members' active engagement in utilizing the LS and OA innovations through implementing change in their classroom practice has had a positive impact on them to rethinking their teaching practice, on their attitudes to students, and about their learning tasks when it comes to improving mathematical reasoning habits, collegiality, and professional selfidentification. This implication is supported by Khokhotva and Albizuri (2020) who found that the LS framework has enormous potential regarding facilitating change in teachers' educational beliefs. These changes are associated with establishing a more positive student-centered teaching approach and encouraging a rights-based school culture in which teachers share their vision and increasing the capacity for innovation. Following this line of reasoning, we suggest that mathematics teachers should be encouraged to use OA treatment in the LS process as a method for improving their students' mathematical reasoning ability.

In conclusion, the results can be rationalized in terms of the current evidence and should be applied in the future to improve students' mathematical reasoning habits from elementary through to high school education levels. This is because researchers discovered that the components of mathematical reasoning habits should be developed through lesson plans across the curriculum. Moreover, the key elements for mathematical reasoning should be included in mathematics content strands. The examples that illustrate the roles of teachers and students in the classroom to reshape the conversation about school mathematics and shift the curriculum toward emphasis on reasoning for all students should be widely introduced to elementary and high school mathematics teachers.

The results might be of value for school leaders, educators, teacher trainers, and policymakers in terms of advocating OA being incorporated into the LS framework as a systematic approach to whole-school enhancement. This would act as a tool to encourage positive change in school culture, as well as give stimulus to future studies involving the school culture perspective about developing LS and OA impact evaluation tools (Khokhotva \& Albizuri, 2020). However, this requires a thorough understanding of the details of LS (Inprasitha, 2011) and OA (Nohda, 2000) concepts, while teachers are planning and implementing OA treatment in the LS process.

\section{ACKNOWLEDGMENT}

This research is supported by Research and Graduate Studies, Khon Kaen University. The authors gratefully acknowledge the use of service and facilities of the Centre for Research in Mathematics Education (CRME), Khon Kaen University, Thailand. 
The contents of this manuscript are derived from the first author's doctoral dissertation thus fulfilling the $\mathrm{Ph}$.D. requirement of Khon Kaen University.

\section{REFERENCES}

Aridor, K., \& Ben-Zvi, D. (2017). The co-emergence of aggregate and modeling reasoning. Statistics Education Research Journal, 16(2), 38-63.

Askew, M. (2020). Reasoning as a mathematical habit of mind. The Mathematical Gazette: A Journal of the Mathematical Association, 104(599), 1-11. https://doi.org/10.1017/mag.2020.1

Baba, T. (2007). Japanese education and lesson study: An overview. In M. Isoda, M. Stephen, Y. Ohara, \& T. Miyakawa (Eds.), Japanese lesson study in mathematics: Its impact, diversity and potential for education improvement (pp. 2-7). World Scientific.

Cuoco, A. I., Goldenberg, P., \& Mark, J. (1996). Habits of mind: An organizing principle for mathematics curriculum. Journal of Mathematical Behavior, 15(4), 375-402.

Doerr, H., Delmas, R., \& Makar, K. (2017). A modeling approach to the development of students' informal inferential reasoning. Statistics Educational Research Journal, 16(2), 86-115.

English, L. (2013). Reconceptualizing statistical learning in the early years. In L. English \& J. Mulligan (Eds.), Reconceptualizing early mathematics learning (pp. 67-82). Springer.

Foster, F. L. (2012). Using reasoning tasks to develop skills necessary to learn independently [Unpublished Doctoral dissertation]. Minot State University.

Hart, L. C., Alston, A. S., \& Murata, A. (Eds.). Lesson study research and practice in mathematics education. Springer Science + Business Media. https://doi.org/10.1007/9718-90-481-9941-9-14
Loipha, S., \& Inprasitha, M. (2004). Development of new teaching profession for enhancing mathematical learning. KKU Journal of Mathematics Education, 1, 18-28.

Inprasitha, M. (2009). Lesson study: Innovation for developing teacher and students [Doctoral thesis]. Khon Kaen University.

Inprasitha, M. (2010). $3^{\text {rd }}$ grade mathematics textbook / Part 1. Center for Research in Mathematics Education (CRME).

Inprasitha, M. (2011). One feature of adaptive lesson study in Thailand: Designing a learning unit. Journal of Science and Mathematics Education in Southeast Asia, 34(1), 47-66.

Inprasitha, M. (2014). Processes of problem solving in school mathematics. Center for Research in Mathematics Education (CRME).

Isoda, M. (2015). The science of lesson study in the problem-solving approach. In M. Inprasitha, M. Isoda, P. Wang-Iverson, \& B. H. Yeap (Eds.), Lesson study: Challenges in mathematics education (pp. 81-108). World Scientific.

Isoda, M., \& Katagiri, S. (2012). The role of questioning in problem-solving approach. In M. Isoda \& S. Katagiri (Eds.), Mathematical thinking: How to develop it in the classroom (pp. 127-128). World Scientific.

Khokhotva, O., \& Albizuri, I. E. (2020). Teachers' educational beliefs change through lesson study: Implications for school culture. International Journal for Lesson \& Learning Studies, 9(4), 317-331.

Kilpatrick, J., Swafford, J., \& Findell, B. (2001). Adding it up: Helping children learn mathematics. National Academy Press.

Lehrer, R., \& English, L. (2018). Introducing children to modeling variability. In D. Ben-Zvi, K. Makar, \& J. Garfield (Eds.), The international handbook of research in statistics education (pp. 229-260). Springer. 
Lewis, C. (2002). Lesson study: A handbook of teacher-led instructional change. Research for Better Schools.

Lim, C. S., Tang, K. N., \& Tan, S. F. (2013). Building rapport with pupils to enhance teaching: Implications from observing three primary excellent teachers. Pertanika Journal of Social Sciences \& Humanities, 21(3), 1009-1022.

Makar, K. (2016). Developing young children's emergent inferential practices in statistics. Mathematical Thinking and Learning, 18(1), 1-24.

Makar, K., \& Rubin, A. (2017). Learning about statistical inference. In D. Ben-Zvi, K. Makar, \& J. Garfield (Eds.), The international handbook of research in statistics education (pp. 229-260). Springer.

Manmai, T., Inprasitha, M., Changsri, N., \& Pattanajak, A. (2020). Development of reasoning habits through lesson study and open approach teaching practices. International Educational Research, 3(2), 29-36.

Martin, G., \& Kasmer, L. (2010). Reasoning and sense making. Teaching Children Mathematics, 16(5), 284-291.

National Council of Teachers of Mathematics. (2000). Principles and standards for school mathematics. National Council of Teachers of Mathematics.

National Council of Teachers of Mathematics. (2009). Focus in high school mathematics reasoning and sense making. National Council of Teachers of Mathematics.

Nohda, N. (2000). Learning and teaching through open-approach method. In Mathematics education in Japan (pp. 29-32). Japan Society of Mathematical Education.

Oslington, G., Mulligan, J., \& Van Bergen, P. (2020). Third-graders' predictive reasoning strategies.
Educational Studies in Mathematics, 104, 5-24. https://doi.org/10.1007/s10649-020-09949-0

Shimizu, Y. (1999). Aspects of mathematics teacher education in Japan: Focusing on teachers' role. Journal of Mathematics Teacher Education, 2(1), 107-116.

Stigler, J. W., \& Hiebert, J. (1999). The teaching gap: Best ideas from the world's teachers for improving education in the classroom. The Free Press.

Stipek, D. J., Givvin, K. B., Salmon, J. M., \& MacGyvers, V. L. (2001). Teachers' beliefs and practices related to mathematics instruction. Teaching and Teacher Education, 17(2), 213-216. https://doi.org/10.1016/S0742-051X(00)00052-4

Tall, D. (2008). Using Japanese lesson study in teaching mathematics. The Scottish Mathematical Council Journal, 38, 45-50.

Tall, D. (2014). Making sense of mathematical reasoning and proof. In Mathematics \& mathematics education: Searching for common ground (pp. 223-235). Springer.

Tan, S. F., Lim, C. S., \& Chew, C. M. (2017). Changes in teachers' reflection after lesson study process. Malaysian Journal of Learning and Instruction, Special Issue, 145-172.

Takahashi, A., \& Yoshida, M. (2004). Ideas for establishing Lesson-Study communities. Teaching Children Mathematics, 426-443.

Thinwiangthong, S., Eddy, C. M., \& Inprasitha, M. (2020). Mathematics teachers' abilities in developing formative assessment after the introduction of lesson study and open approach innovations. Malaysian Journal of Learning and Instruction, 17(1), 101-132.

Wagner, T. (2008). The global achievement gap: Why even our best schools don't teach the new survival skills our children need - and what we can do about it. Basic Books. 
Zhao, Y. (2009). Catching up or leading the way: American education in the age of globalization. Association for Supervision and Curriculum Development. 